\title{
Evaluating insole design with joint motion, plantar pressure and rating of perceived exertion measures ${ }^{1}$
}

\author{
Yu-Chi Lee ${ }^{\text {a }}$, Gloria Lin ${ }^{\text {a }}$, Mao-Jiun J. Wang ${ }^{\text {a,* }}$ \\ ${ }^{a}$ Department of Industrial Engineering and Engineering Management, National Tsing Hua University, Hsinchu, \\ Taiwan, $R O C$
}

\begin{abstract}
This study compared a newly-designed insole with two commercially available insoles. The response measures included joint motion, plantar pressure and rating of perceived exertion. Thirty healthy female students were paid to participate in this study. The results showed that significant differences among the three insoles were found in plantar pressure and rating of perceived exertion. There were no significant differences among three different insoles in join motion measures. When wearing the newly-designed insole, the plantar pressure under heel area would transfer to midfoot area, and resulted in lower subjective discomfort ratings in midfoot and heel areas. The barefoot condition showed the worst performances in most of the response measures. The findings of this study provide very useful information for ergonomic insole design.
\end{abstract}

Keywords: insole, joint motion, plantar pressure, rating of perceived exertion

\section{Introduction}

Insoles with different design tend to affect the gait performances. Heel cup and arch support are two important features for insoles design. Ergonomic heel cup design can reduce foot pressure in heel area [8] and adequate arch support design can distribute plantar pressure evenly [4]. In addition, using an ergonomic insole can decrease the occurrence of plantar fasciitis [2].

Cushion design is another approach to reduce foot plantar pressure. Hinz et al. [7] evaluated the effectiveness of cushioning insole on load reduction in comparison with conventional insoles. The results showed that cushioning insole had better performance on load reduction under metatarsal area. Hsi et al. [9] noted that using the cushion pad can decrease the foot pressure, but the position of cushion pad is another important factor. Further, Tsai et al. [10] suggested that using the different insole material in different region can increase wearing comfort and distribute the foot pressure evenly. Moreover, Creaby et al. [6] compared three different insoles (no insole, flat material insole and heel-cup insole) on impact loading during walking and indicated that peak impact force at the knee was reduced with flat material insoles and heel-cup insoles. Chung et al. [5] evaluated the effect of insole with the combination of different insole designs (e.g. heel cup, arch support and cushion) on gait performance and reported that different insole designs and materials showed different influence on gait performance. Further, Basford and Smith [3] investigated that the effectiveness of insoles in reducing lower limbs pain and concluded that using insoles can effectively improve comfort and reduce back, leg and foot pain for individuals who must stand throughout the day.

In semiconductor industry, people who work in clean room environment tend to have prolonged walking and standing. Lin et al. [1] evaluated the different clean room boots on biomechanical, physiological and psychophysical responses and reported

\footnotetext{
${ }^{1}$ Evaluating insole design

*Mao-Jiun J. Wang. E-mail: mjwang@ie.nthu.edu.tw
} 
that the shock absorption of the boot had significant effects on selected measurements. But information about the effect of insole with clean room boots is still lacking. It would be interesting to investigate the effect of placing insole in clean room boots. Therefore, this study aims to evaluate a new insole with high medial heel cup and correct arch support being placed in clean room boots through the measures of joint motion, plantar pressure, and rating of perceived exertion (RPE).

\section{Method}

\subsection{Participants}

Thirty healthy female students were paid to participate in this study. The mean age was $21.9( \pm 1.6)$ years, the mean body height was $160.1( \pm 5.8) \mathrm{cm}$ and the mean body weight was $52.2( \pm 6.2) \mathrm{kg}$. The mean foot length and foot breadth of the participants were $23.5( \pm 1.0) \mathrm{cm}$ and $9.4( \pm 0.5) \mathrm{cm}$ respectively. None of them has any musculoskeletal disorders history or lower limbs problems.

\subsection{Apparatus and material}

Three different insoles being placed in clean room boots were evaluated. The three insoles included a newly-designed insole, adjustable-arch-support insole, and ergo-design insole. In order to simulate the clean room workers in semiconductor industry, barefoot wearing the clean room boots without insole was also selected. The characteristics of the three insoles are shown in Table 1.The response measures included joint motion, plantar pressure, and rating of perceived exertion. A motion capture system (Proreflx MCU 240, Qualisys, Sweden) with six digital cameras was applied to capture the joint angle of pelvic, hip, knee, and ankle. Plantar foot pressure data were collected by using a scan system (F-Scan ${ }^{\circledR}$ System, Tekscan, USA). Seven zones of plantar pressure including the hallux, 2-5th phalanges, 1st metatarsal, 2-3th metatarsal, 4-5th metatarsal, midfoot and heel zones were measured. The peak pressure of each zone was recorded. Borg CR-10 rating scale was used to assess the perceived exertion of foot and plantar area, including ankle, instep, forefoot, hallux, 2-5th phalanges, metatarsal, arch, and heel. Borg CR-10 is a 10-point psychophysical assessment scale, while score 10 for "maximal pain" and 0 for "nothing at all".

\subsection{Procedure}

Before data collection, each participant was given information about the purpose and procedure of this experiment. The demographic data and anthropometric data were collected. Thirty-seven markers were placed on the important anatomical landmarks on both side of body. The markers positions included heel, metatarsal, ankle, knee, hip and pelvis. The four insole conditions were randomly assigned. For adjustable-arch-support condition, participants were asked to adjust the arch pad to a comfortable position. Each testing insole was inserted into a clean room boots before wearing.

Table 1. The characteristics of the three insoles in this study

\begin{tabular}{lccc}
\hline Clean room boots & Adjustable-arch-support insole & New-design insole \\
& & & \\
\hline Material & & & \\
Weight & $44 \mathrm{~g}$ & $50 \mathrm{~g}$ & PU \\
Breadth & $83.9 \mathrm{~mm}$ & $85.5 \mathrm{~mm}$ & $53 \mathrm{~g}$ \\
Thickness & Forefoot: $3.6 \mathrm{~mm}$ & Forefoot: $6.6 \mathrm{~mm}$ & $90.8 \mathrm{~mm}$ \\
& Heel area: $7.0 \mathrm{~mm}$ & Feel area: $10.0 \mathrm{~mm}$ & Heefoot: $4.6 \mathrm{~mm}$ \\
Arch support & Yes $($ soft & Yes (correctly position) \\
Arch support height & Yes (adjustable pad) & $27.0 \mathrm{~mm}$ \\
Forefoot cushion & $16.5 \mathrm{~mm}$ & Yes & Yes \\
Heel cushion & Yes & Yes & Yes \\
Heel cup & Yes & Medial: $23.2 \mathrm{~mm}$ & Medial: $21.3 \mathrm{~mm}$ \\
& Medial: $20.0 \mathrm{~mm}$ & Lateral: $23.2 \mathrm{~mm}$ & Lateral: $10.9 \mathrm{~mm}$ \\
\hline
\end{tabular}


During the experiment, all participants were requested to walk on a treadmill for 20 min with walking speed at $3 \mathrm{~km} / \mathrm{hr}$.A metronome was used to pace the walking rhythms for each participant. Then, each participant was asked to walk on an 8-m walkway (1.2 m width path) with the same walking speed and rhythms. Joint motion and plantar pressure were collected when the participants walked through the center of walkway. Each condition was repeated five times. After walking task, participants had to complete the subjective discomfort rating questionnaire by using Borg CR-10 scale. A least 10 min rest time was given between the two experiment trails to avoid fatigue effect.

\subsection{Statistical analysis}

One-way ANOVA was conducted to evaluate the insole effect on the response measures. The dependent variables included joint range of motion, plantar pressure and subjective discomfort rating. Duncan's multiple rage test was also used as pos-hoc testing.

\section{Results and discussion}

\subsection{Join motion}

Table 2 shows that the mean and standard deviation of lower extremity joint motion in sagittal plane and coronal plane. The ANOVA results indicated that walking with the three different insoles showed no influence on the join motion. In general, heel cup design was to increase the walking stability. The medial height was equal to lateral height of heel cup design. In here, the slope of heel cup of the newlydesigned insole was based on the 3D scanning data, which the heel cup height in medial side was higher than in lateral side. Since, the newly-designed insole with higher medial heel cup showed no effect on joint motion, the insole with lower lateral height of heel cup did not show influences on the join motion during walking.

\subsection{Plantar pressure}

The ANOVA results in Table 3 showed that there was significant insole effect plantar pressure in hallux, 2-5th phalanges, 2-3th metatarsal, midfoot and heel areas. Further, Duncan's MRT revealed that the newly-designed insole had the smallest plantar pressure in hallux $(71.8 \mathrm{~N}), 2-5$ th phalanges $(76.5 \mathrm{~N})$ and heel area (104.3 N). Generally, a good arch support design should be able to distribute the plantar pressure evenly [4]. The newly-designed insole was able to redistribute the plantar pressure from forefoot and heel area to midfoot area. Comparing the results of the newly-designed insole with barefoot condition, the smaller plantar pressure was found while wearing the newly-designed insole. It means that the arch support design of the newly-designed insole can effectively support arch and to distribute the plantar pressure evenly.

\subsection{Rating of perceived exertion}

The means of the rating of perceived exertion (RPE) are shown in Table 4. Significant insole effect was found in the RPE of the midfoot and heel areas (as shown in Table 4). From the Duncan's post-hoc test results, while wearing the newly-designed insole, a better performance in RPE in the heel area was observed. Kogler et al. [2] indicated that the higher plantar pressure will increase the occurrence of plantar fasciitis. When wearing the newly-designed insole, the plantar pressure under heel area would transfer into midfoot area, and the subjects' comfortable response in midfoot area while walking with the newlydesigned insole.

Moreover, the adjustable-arch-support insole had the best RPE than the other two insoles in midfoot area. The adjustable-arch-support insole had adjustable arch support pad. The Duncan's MRT result showed that there was no significant difference in RPE between adjustable-arch-support insole and newly-designed insole in midfoot area. It indicated that the arch support position of the newly-designed insole was adequate. On the other hand, the barefoot condition showed the worst RPE scores in all the foot and ankle areas.

\section{Conclusions}

This study compared the newly-designed insole with two commercially available insoles on join motion, plantar pressure and subjective discomfort ratting. No significant insole effect on join motion was found. For plantar pressure measure, the newlydesigned insole showed better performances in hallux, 2-5th phalanges, heel area. For RPE responses, subjects responded the higher RPE scores in midfoot area when wearing newly-designed insole. The newly-designed insole can transfer the plantar pressure 
from heel to midfoot and distribute the pressure evenly, and resulted in a lower RPE in heel area. The find- ings of this study provide very useful information for designing an ergonomic insole.

Table 2. The ANOVA results of join motion (unit in degree)

\begin{tabular}{|c|c|c|c|c|c|c|}
\hline & Item & Barefoot & $\begin{array}{l}\text { Adjustable-arch- } \\
\text { support insole }\end{array}$ & Ergo-design insole & $\begin{array}{c}\text { Newly-designed } \\
\text { insole }\end{array}$ & p-value \\
\hline \multirow{2}{*}{ Pelvic } & Max pelvic obliquity & $2.85(5.15)^{+}$ & $2.56(4.11)$ & $2.45(5.15)$ & $2.80(4.91)$ & NS \\
\hline & Min pelvic obliquity & $-5.36(3.98)$ & $-5.29(3.12)$ & $-5.33(3.47)$ & $-5.16(4.45)$ & NS \\
\hline \multirow{2}{*}{ Hip } & Max hip adduction & $10.76(3.55)$ & $10.47(2.54)$ & $10.55(3.02)$ & $10.84(4.66)$ & NS \\
\hline & Min hip adduction & $-1.75(6.15)$ & $-1.91(3.38)$ & $-2.15(4.11)$ & $-1.52(5.90)$ & NS \\
\hline \multirow{2}{*}{ Knee } & Max knee flexion & $28.89(4.34)$ & $29.53(4.25)$ & $29.82(4.84)$ & $30.09(6.20)$ & NS \\
\hline & Min knee flexion & $4.21(-4.09)$ & $2.93(-3.08)$ & $2.74(-2.59)$ & $2.19(-1.95)$ & NS \\
\hline \multirow{2}{*}{ Ankle } & Max ankle flexion & $15.49(4.31)$ & $15.24(3.57)$ & $14.11(4.57)$ & $14.47(4.48)$ & NS \\
\hline & Min ankle flexion & $-11.71(3.33)$ & $-9.79(3.45)$ & $-10.84(0.76)$ & $-10.49(1.55)$ & NS \\
\hline
\end{tabular}

Table 3. The ANOVA results of plantar pressure (unit in $\mathrm{N}$ )

\begin{tabular}{|c|c|c|c|c|c|c|c|}
\hline Item & Hallux & $2-5^{\text {th }}$ phalanges & $1^{\text {st }}$ metatarsal & $2-3^{\text {th }}$ metatarsal & $4-5^{\text {th }}$ metatarsal & Midfoot & Heel \\
\hline Barefoot & $172.0(40.2)^{+} \mathrm{A}^{-}$ & $251.1(37.2) \mathrm{A}$ & $163.6(43.2)$ & $323.1(30.9) \mathrm{A}$ & $325.3(54.3)$ & $236.9(57.9) \mathrm{A}$ & $181.0(31.1) \mathrm{A}$ \\
\hline $\begin{array}{l}\text { Adjustable-arch- } \\
\text { support insole }\end{array}$ & $120.2(24.3) \mathrm{A}$ & $132.5(30.0) \mathrm{B}$ & $177.9(38.7)$ & $252.0(40.6) \mathrm{AB}$ & $370.4(54.6)$ & $123.8(33.7) \mathrm{AB}$ & $133.7(30.2) \mathrm{AB}$ \\
\hline Ergo-design insole & $102.0(20.1) \mathrm{A}$ & $81.8(30.1) \mathrm{B}$ & $165.3(33.9)$ & $165.0(29.3) \quad$ B & 343.1 (15.7) & $98.6(25.1)$ & $106.7(24.8) \mathrm{B}$ \\
\hline $\begin{array}{l}\text { Newly-designed } \\
\text { insole }\end{array}$ & 71.8 (15.4) B & $76.5(36.5) \mathrm{B}$ & $168.3(30.8)$ & $238.1(39.8) \mathrm{AB}$ & $355.3(54.0)$ & 185.9 (31.0) AB & 104.3 (22.6) B \\
\hline p-value & * & $*$ & NS & $*$ & NS & $*$ & * \\
\hline
\end{tabular}

Table 4. The ANOVA results of RPE scores

\begin{tabular}{cccccccccc}
\hline Item & Ankle & Instep & Forefoot & Hallux & $2-5^{\text {th }}$ phalanges & Metatarsal & Midfoot & Heel \\
\hline Barefoot & 1.23 & 2.37 & 3.07 & 2.13 & 2.57 & 3.07 & $3.00 \mathrm{~A}^{-}$ & $3.67 \mathrm{~A}$ \\
$\begin{array}{c}\text { Adjustable-arch- } \\
\text { support insole }\end{array}$ & 0.97 & 1.13 & 2.03 & 1.27 & 1.07 & 1.87 & $1.03 \mathrm{~B}$ & 1.77 & $\mathrm{~B}$ \\
$\begin{array}{c}\text { Ergo-design insole } \\
\text { Newly-designed }\end{array}$ & 1.00 & 1.50 & 1.73 & 1.37 & 1.67 & 1.00 & 2.00 & $1.43 \mathrm{~B}$ & $2.20 \mathrm{AB}$ \\
$\begin{array}{c}\text { insole } \\
\text { p-value }\end{array}$ & 1.00 & 1.03 & 1.27 & 1.10 & $\mathrm{NS}$ & $\mathrm{NS}$ & $*$ \\
\hline
\end{tabular}

: Duncan's post-hoc test; *: $\mathrm{p}<0.05 ;$ NS: not-significant

\section{References}

[1] C. L. Lin, M. J. Wang and C. G. Drury, Biomechanical, physiological and psychophysical evaluations of clean room boots, Ergonomics, 2007, 50, pp. 481-496.

[2] G. F. Kogler, S. E. Solomonidis and J. P. Paul, Biomechanics of longitudinal arch support mechanisms in foot otthoses and their effect on plantar aponeurosis strain, Clinical Biomechanics, 1996, 11, pp. 243-252.

[3] J. R. Basford and M. A. Smith, Shoe insoles in the workplace, Orthopedics, 1988, 11, pp. 285-288

[4] M. C. Chiu and M. J. Wang, Professional footwear evaluation for clinical nurses, Applied Ergonomics, 2007, 38 (2), pp. 133-141.

[5] M. J. Chung, C. L. Lin, C. H. Chang and M. J. Wang, A comparison of different shoes insoles in gait performance, the 3 th International Conference on Applied Human Factors and Ergonomics, 2010.
[6] M. W. Creaby, M. Kirsty and L. B. Kim, Insole effects on impact loading during walking, Ergonomics, 2011, 54 (7), pp. 665-671.

[7] P. Hinz, A. Henningsen, G. Matthes, B. Jäger, A. Ekkernkamp and D. Rosenbaum, Analysis of pressure distribution below the metatarsals with different insoles in combat boots of the German Army for prevention of march fractures, Gait \& Posture, 2008, 27, pp. 535-538.

[8] S. Goske, A. Erdemir, M. Petre, S. Budhabhatti, and P. R. Cavanagh, Reduction of plantar heel pressures: Insole design using finite element analysis, Journal of Biomechanics, 2006, 39, pp. 2363-2370

[9] W. L. Hsi, J. H. Kang and X. X. Lee, Optimum position of metatarsal pad in metatarsalgia for pressure relief, American Journal of Physical Medicine \& Rehabilitation, 2005, 84 (7), pp. 514-520.

[10] Y. C. Tsai, S. L. Chang, S. W. Yang and S. M. Lai, Effect of insole and sock materials on walking plantar pressure in diabetic patients, Journal of Biomechanics, 2007, 40, S448. 